\title{
Muséologies
}

Les cahiers d'études supérieures

muséologies

\section{Variabilité, identité spécifique et numérique des oeuvres contemporaines}

\section{Francine Couture}

Volume 5, numéro 1, automne 2010

URI : https://id.erudit.org/iderudit/1033525ar

DOI : https://doi.org/10.7202/1033525ar

Aller au sommaire du numéro

\section{Éditeur(s)}

Association Québécoise de Promotion des Recherches Étudiantes en Muséologie (AQPREM)

\section{ISSN}

1718-5181 (imprimé)

1929-7815 (numérique)

Découvrir la revue

Citer cet article

Couture, F. (2010). Variabilité, identité spécifique et numérique des oeuvres contemporaines. Muséologies, 5(1), 138-177. https://doi.org/10.7202/1033525ar

\section{Résumé de l'article}

La réexposition d'oeuvres contemporaines sous la forme de la présentation de nouveaux exemplaires physiques d'une oeuvre conçue pour être unique questionne les notions de variabilité, d'identité spécifique et numérique de l'oeuvre d'art. Dans cet article, Couture étudie trois cas de figure propres à cette réalité : la production d'une réplique ou d'une copie d'exposition, l'exposition d'un élément d'une oeuvre, non pas comme un fragment, mais comme une oeuvre autonome, et enfin l'exemple particulier de l'actualisation d'un énoncé verbal qui est porteur des valeurs d'unicité et de singularité attribuées à l'oeuvre d'art. Elle conclut qu'il en résulte une transformation de l'institution muséale et de l'artiste engendrant notamment une redéfinition des rôles de chacun. 
Francine Couture

\section{Variabilité, identité spécifi- que et numérique des œuvres contemporaines}

La réexposition d'œuvres contemporaines sous la forme de la présentation de nouveaux exemplaires physiques d'une œuvre conçue pour être unique questionne les notions de variabilité, d'identité spécifique et numérique de l'œuvre d'art. Dans cet article, Couture étudie trois cas de figure propres à cette réalité : la production d'une réplique ou d'une copie d'exposition, l'exposition d'un élément d'une œuvre, non pas comme un fragment, mais comme une œuvre autonome, et enfin l'exemple particulier de l'actualisation d'un énoncé verbal qui est porteur des valeurs d'unicité et de singularité attribuées à l'œuvre d'art. Elle conclut qu'il en résulte une transformation de l'institution muséale et de l'artiste engendrant notamment une redéfinition des rôles de chacun.

Francine Couture est professeure au Département d'histoire de l'art de l'Université du Québec à Montréal. Ses travaux de recherche en cours portent sur les rapports de l'art contemporain avec l'institution artistique. Elle dirige le projet de recherche intitulé "Réexposition, réactualisation et pérennité des œuvres contemporaines" Elle a coordonné la publication des livres suivants: Les arts visuels au Québec dans les années soixante, La reconnaissance de la modernité (VLB éditeur): Les arts visuels au Québec dans les années soixante, L'éclatement du modernisme (VLB éditeur) et Exposer rart contemporain du Québec (Centre de diffusion 3D). coUTURE.FRANCINE@UQAM.CA 


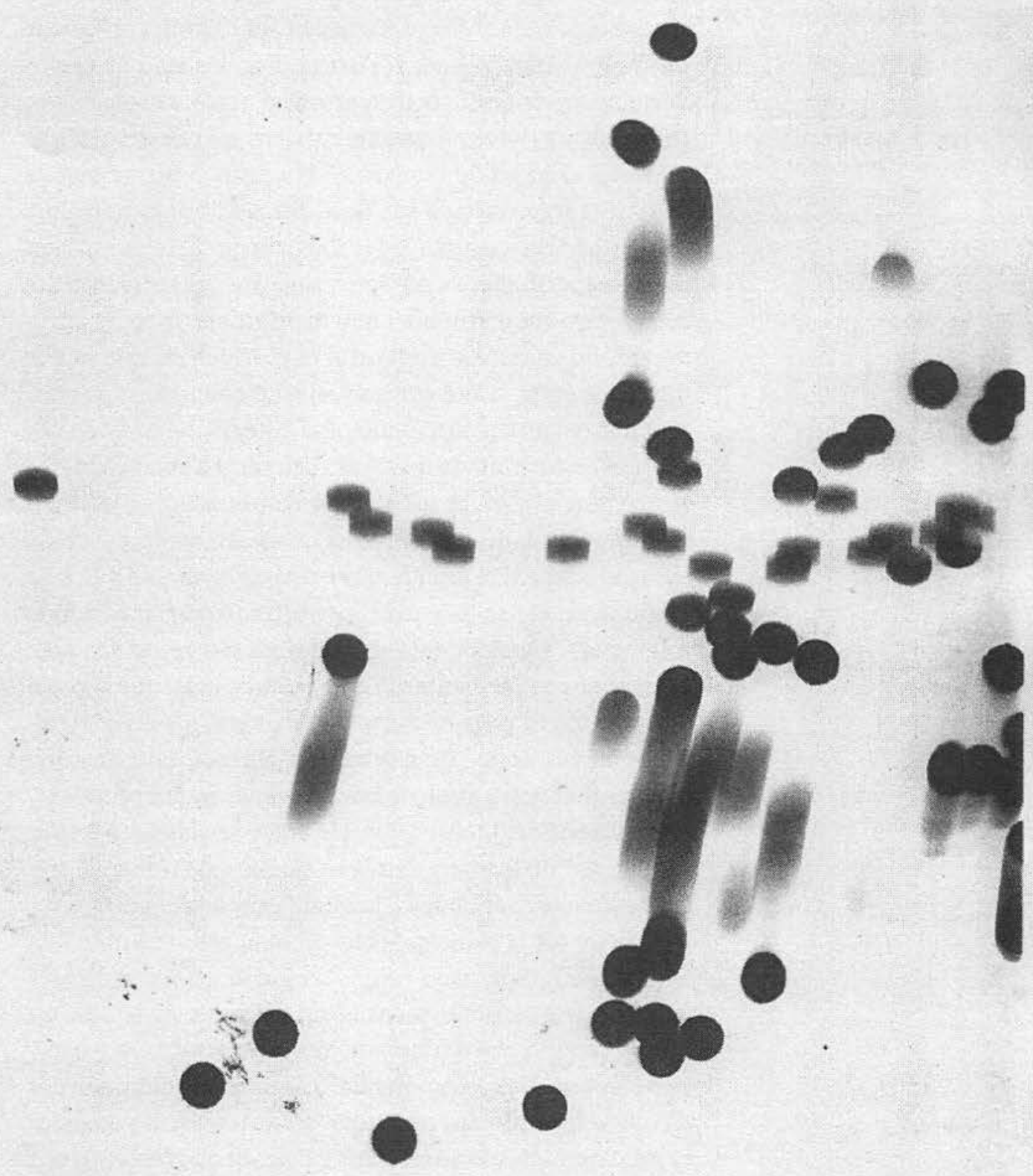


1

PRIETO, Luis. "Le mythe de l'original. L'original comme objet d'art et comme objet de collection ». In. GENETTE, Gérard (dir.). Esthétique et Poétique. Paris: Seuil, 1992, p. 137-156. 2

GENETTE, Gérard. L'ceuvre de l'art Immanence et transcendance. Paris: Seuil, 1994, p. 188.

3 Id., p. 247.

4

TATE MODERN GALLERY. Inherent Vice: The Replica and its Implication in Modern Sculpture, 18-19 octobre 2007 Tate Research, $n^{2} 8$. <http://www. tate.org.uk/research/tateresearch/ tatepapers/o7automn/> (consulté en mars 2010).
La réexposition d'œuvres contemporaines génère souvent des variations qui se manifestent sous divers modes. Une nouvelle occurrence peut entraîner la modification des composantes sans pour autant donner lieu à un nouvel exemplaire, mais elle peut aussi produire un nouvel exemplaire d'une œuvre qui avait été conçue pour être unique. Cette situation met en cause les conventions de l'institution muséale dont les pratiques et les discours, relevant du système traditionnel des beaux-arts, visent à préserver l'originalité et l'unicité des œuvres d'art de sa collection. Le musée s'est donné comme mission de préserver l'état matériel originel de l'œuvre d'art originale parce qu'elle constitue une trace de son acte de création ou renvoie à cette expérience singulière de l'artiste qui lui a donné son identité spécifique. Luis Prieto considère que cette identité spécifique, qui distingue l'œuvre originale de toute autre œuvre, lui confère une identité numérique ou une valeur d'unicité et de rareté sur lesquelles est fondée sa valeur, tant marchande qu'artistique'. Par ailleurs, Gérard Genette postule qu'une œuvre originale peut être l'objet d'immanence plurielle ou se manifester en plusieurs objets non identiques et concurrents ${ }^{2}$, sans nécessairement mettre en cause son caractère unique. Par exemple, souligne-t-il, une réplique est considérée par l'institution artistique comme une nouvelle version de l'œuvre originale qui se situe en rapport de complémentarité avec celle-ci, alors que la copie d'exposition de cette même œuvre en constitue une "manifestation indirecte", car elle a été, le plus souvent, produite pour pallier son "absence définitive ou momentanée " 3 . Cette classification est toutefois objet de réflexion à l'intérieur de l'institution muséale. Quelle valeur et quel statut doivent être accordés à la réplique et à la copie d'exposition? Ce fut la principale question du colloque tenu à la Tate Modern Gallery en $2007^{4}$. Les participants ont relevé qu'il n'existe pas de consensus qui permette de définir un protocole servant à donner une seule réponse à cette question; ils ont alors recommandé la définition commune des termes d'un ordre hiérarchique servant à différentier les types d'exemplaires se référant à l'œuvre originale. Ils ont cependant distingué la réplique produite par l'artiste de la copie d'exposition réalisée par le restaurateur ou le techni- 
cien du musée. Considérant la réplique comme une nouvelle version de l'œuvre originale, dotée d'une nouvelle date de production, ils lui ont reconnu une pleine valeur artistique. Alors qu'ils ont fait valoir la fonction informative de la copie d'exposition en lui attribuant le statut de document ; ils ont noté que le nouvel exemplaire, dont la production est autorisée par les ayants droit de l'artiste, a aussi ce statut de document et non celui d'une nouvelle version de l'œuvre originale.

Notre analyse empirique de production de répliques et de copies d'exposition a fait ressortir une manipulation de cette classification qui résulte le plus souvent de négociations entre l'artiste ou ses ayants droits et l'institution muséale. Nous porterons notre attention sur ces négociations ainsi que sur les stratégies mises en œuvre par le musée pour maintenir les valeurs d'originalité et d'unicité de l'œuvre acquise.

Nous analyserons également le cas de figure de réexposition d'œuvres ayant donné lieu à la présentation d'un ou de plusieurs éléments d'une œuvre déjà produite comme œuvre autonome et non pas comme un fragment ou une version réduite de l'œuvre originale. Nous considérons que ce cas de figure interroge la représentation que l'artiste et le collectionneur se font de ces œuvres et des éléments qui les constituent, ainsi que les modalités de reconnaissance de leur intégralité, tant matérielle que conceptuelle.

Enfin, nous examinerons comment l'institution muséale a établi un cadre de gestion d'œuvres contemporaines acquises sur le mode de l'énoncé verbal ou qui doivent être réactualisées à chacune de leur prestation. Ces œuvres relèvent du régime des arts allographiques dont le propre, écrit Cérard Cenette, est de "permettre une multiplication illimitée des exemplaires de manifestations d'un objet d'immanence idéal et unique ${ }^{5}$. A la différence des cas de figure précédemment mentionnés, ces divers exemplaires ne mettent donc pas en cause l'identité numérique et spécifique d'une œuvre originale dotée d'une matérialité, car ils résultent de l'actualisation de prescriptions transmises par une
5

GENETTE, Gérard. L'œuure de l'art Immanence et transcendance. Paris: Seuil, 1994, p. 200. 
6

Nous empruntons cette notion à Didier SEMIN (Le Peintre et son modèle. Cenève: Mamco, 2001) qui s'est référé aux écrits de Nelson Goodman et de Cérard Genette: le script, écrit-il, est tout document utilisant une écriture qui fixe un scénario pouvant être écrit, mais aussi dessiné et photographié, servant à guider la réinstallation des œuvres et à préserver leur mémoire ou leur pérennité.

7

GUELTON, Bernard. L'exposition, interprétation et réinterprétation. Paris: L'Harmattan, coll. "Ouverture philosophique», 1998, p. 57.

8

Archives de conservation, Musée des beaux-arts du Canada. Lettre de Robert Morris à Brydon Smith, 14 mars 1970.

9

MORRIS, Robert. "Anti-form ", Artforum 6, avril 1968, p. 33-35.

10

Archives de conservation, Musée des beaux-arts du Canada. Lettre de Mrs. Coates (Galerie nationale du Canada) à Miss Martha Morris (Corcoran Gallery), 15 septembre 1970. notation ou un script ${ }^{6}$ qui consigne les éléments constitutifs de cette ceuvre idéale, laquelle est unique et singulière. Bernard Guelton souligne avec justesse que cette catégorie a opéré un déplacement de la matérialité originelle de l'œuvre vers son auteur en introduisant l'idée que, par la médiation de ce script, l'authenticité de sa prestation procède du jugement de l'artiste qui en fixe ainsi les conditions ${ }^{7}$. La détention d'une telle autorité peut aussi le ou la conduire, ou encore inciter ses ayants droit, à modifier l'identité spécifique d'une œuvre allographique en accordant à l'un de ses exemplaires une pérennité matérielle. Nous analyserons également un cas de figure de ce mode de transformation.

\section{Réplique et copie d'exposition}

Abordons l'analyse de cas de figure d'immanence plurielle d'œuvres originales de collections muséales sur le mode de la réplique et de la copie d'exposition qui ont incité leurs propriétaires à définir un cadre de conduite afın d'établir le statut de ces nouveaux exemplaires.

Le premier cas est celui de Untitled (1967-1968) de Robert Morris, une œuvre acquise en 1969 par le Musée des beauxarts du Canada (ill. 1). Après avoir été prêtée à la Corcoran Gallery of Art (Washington), elle est revenue à son propriétaire amputée de 55 morceaux. Informé de cette situation par le conservateur Brydon Smith, Robert Morris a décidé de fabriquer un nouvel exemplaire parce que le feutre ayant été utilisé pour produire l'œuvre originale n'était plus disponible sur le marché; il ne pouvait donc refaire les morceaux manquants. Il a alors employé un feutre d'une couleur et d'une épaisseur différentes ${ }^{8}$. On peut expliquer cette décision par le fait que Morris avait développé une démarche artistique reconnaissant que l'authenticité d'une œuvre est fondée sur son identité conceptuelle et sur le respect du processus de sa réalisation plus que sur l'intégrité de son matériau originel ${ }^{9}$. La facture qu'il a soumise à la Corcoran Gallery témoigne de cette prise de position, car elle ne couvre que les frais de matériaux et de fabrication et n'affiche pas la valeur marchande de l'œuvre ${ }^{10}$, ce qui indi- 
que que Morris n'a pas accordé au deuxième exemplaire une identité spécifique distincte de l'œuvre originelle.

Le Musée des beaux-arts du Canada possède donc deux versions de Untitled (1967-1968). Il y a l'exemplaire original, qui peut être qualifié de version lacunaire à cause de son état incomplet", et la réplique, produite entièrement par l'artiste, dont la couleur et l'épaisseur du matériau la différentient de l'exemplaire original. Le Musée des beaux-arts du Canada n'a pas donné à cette réplique un nouveau numéro d'acquisition, mais il lui a attribué une nouvelle date de production et l'a classée comme copie de prêt, laissant ainsi entendre qu'il lui accorde un statut hybride conjuguant celui de l'œuvre originale et du document et qu'il lui accorde une identité numérique distincte de l'exemplaire original. Toutefois, afin de préserver la valeur de rareté du premier exemplaire, il interdit l'exposition simultanée des deux exemplaires et n'autorise que la présentation de la version lacunaire dans ses salles ${ }^{12}$. Cette prescription, visant à préserver la valeur de référence de l'exemplaire original à son acte de création, s'accorde avec les propos tenus au colloque de la Tate Gallery, précédemment mentionné, à l'effet que la fabrication d'un deuxième exemplaire d'une œuvre, conçue pour être unique, génère une perte de son aura et de sa référence au passé.

La préservation de l'unicité et de la valeur historique des œuvres de sa collection constitue donc un principe que le musée n'enfreint pas facilement. La production d'un deuxième exemplaire de Salle blanche (1975) de Marcel Broodthaers, acquise en 1989 par le Musée national d'art moderne (Centre Pompidou, Paris) (MNAM), constitue un cas de figure d'une situation inédite qui a obligé cette institution muséale à développer un nouveau cadre de conduite tout en protégeant la valeur de rareté de cette œuvre de sa collection (ill, 2).

Marcel Broodthaers a créé Salle blanche dans le cadre de l'exposition L'Angelus de Daumier présentée au Centre national d'art contemporain à l'automne 1975. Cette œuvre se réfère à une pièce de la maison de l'artiste à Bruxelles où, en 1968,
11

GENETTE, op.cit. p. 240.

12

Archives de conservation, Musée des beaux-arts du Canada. "Check List Loan" adressée à la South Bank Center pour l'exposition Gravity and Grace. The Changing Condition of Sculpture 1965-1975. On peut y lire que la version qui voyage est la version fabriquée en 1970 . 


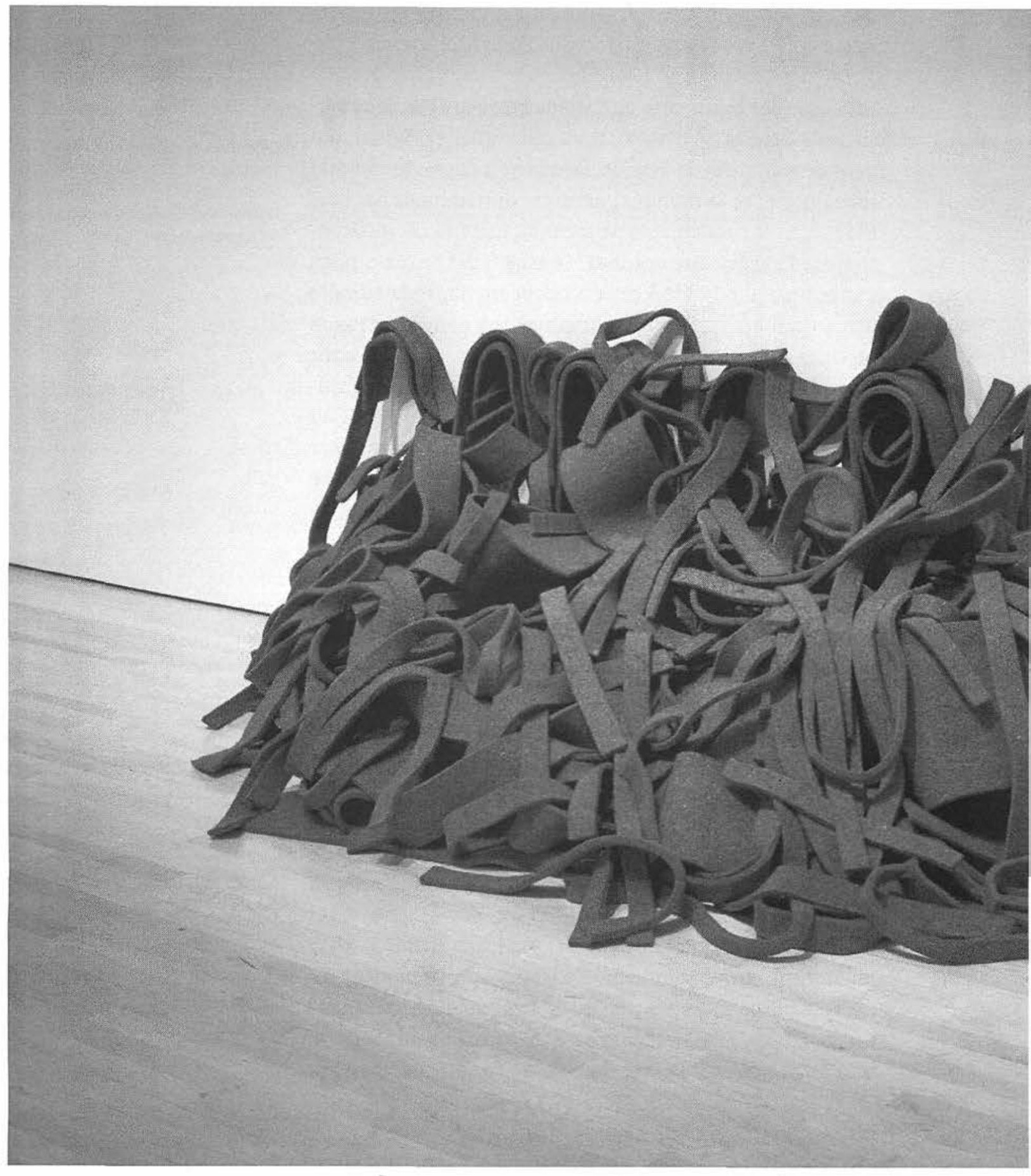




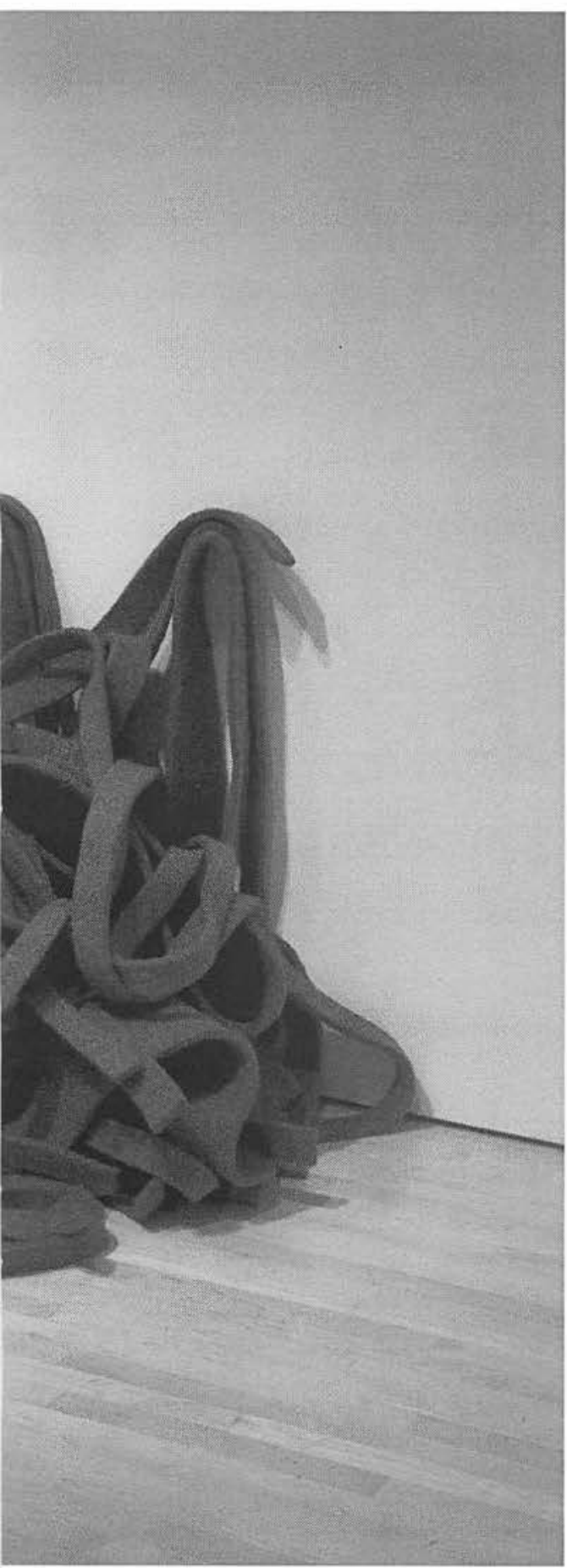

\section{Illustration 1}

Morris, Robert (1931--), Untitled, 1967-1968, 254 morceaux de feutre. Collection Musée des Beaux-Arts du Canada. Crédit photo: $\odot$ Musée des beaux-arts du Canada. $\odot$ Robert Morris / SODRAC Montréal 2010 


\section{Illustration 2}

Broodthaers, Marcel (1924-1976),

OARS, NY, Salle blanche, 1975, bois, avec inscriptions peintes, photographies et ampoules, $390 \times 336 \times$ $658 \mathrm{~cm}$. AM 198. Collection Musée national d'art moderne, Centre Pompidou, Paris, France. Crédit photo: CNAC/MNAM/Dist. Réunion des Musées nationaux / Art Resource, NY 





13

GOUDINOUX, Véronique. "Voguez à ma suite camarade aviateurs, Prologue à une exposition", 50 espèces d'espace, cuvres du Centre Ceorges-Pompidou Musée national d'art moderne. Paris: Editions du Centre Pompidou, 1998, p. 22. 14

A titre d'exemple, mentionnons la copie présentée à la rétrospective des œuvres de Broodthaers au Walker Art Center en 1989. La légende accompagnant la photographie de l'œuvre publiée dans The Burlington Magazine mentionne que la copie a été fabriquée par Jacques Gaumont et jennifet Gough Cooper. (HAXTHAUSEN, Charles W. "Marcel Broodthaers. Los Angeles". The Burlington Magazine, vol. 131, ne 1039 . 1989. p. 739.)

15

Archives de collection, Musée national d'art moderne (Centre Pompidou). Lettre d'Alfred Pacquement à Dominique Bozo, 13 juin 1991. Par exemple, cet exemplaire a été présenté lors des expositions suivantes: Marcel Broodthaers, Galerie nationale du Jeu de Paume, décembre 1991 - mars 1992; 22 Biennale de Sao Paulo, 1994; Les années 70, l'art en cause, CAPC MAC Bordeaux, 18 octobre 2002 - 19 mai 2003: Berlin-Moskau / Moskau - Berlin 1950-2000, Martin Gropius Bau, 2003-2004. il avait ouvert un musée fictif - Le Musée d'Art Moderne, Département des Aigles, Section XIXe siècle. Sa reconstitution n'est toutefois pas identique: au lieu d'y présenter les objets de la pièce originale, l'artiste a écrit sur les murs des mots renvoyant au monde de l'art, au musée et aux procédés et techniques traditionnels de la peinture's.

Depuis son insertion dans la collection du MNAM, Salle blanche a été l'objet de refabrications. Lorsqu'elle a été exposée à l'extérieur de ses murs, l'institution a procédé à la fabrication d'une copie d'exposition ${ }^{14}$. Par ailleurs, au début des années 1990, un deuxième exemplaire fut produit sous la supervision de l'ayant droit de l'artiste, Marie Glisser-Broodthaers, qui voulait ainsi accroître les possibilités de circulation et d'exposition de l'œuvre. Le statut attribué à cet exemplaire par l'institution muséale et l'ayant droit conjugue, comme dans le cas de Untitled (1967-1968) de Robert Morris, les caractéristiques de la réplique et de la copie d'exposition. D'une part, comme dans le cas d'une réplique, une identité numérique distincte lui est reconnue, car il est pérenne et il demeure la propriété de Marie Glisser-Broodthaers. Mais, d'autre part, lui est aussi attribué le statut de copie de voyage dont le protocole établi par le MNAM a fixé les modalités de présentation. Les propriétaires respectifs des deux exemplaires de Salle blanche sont liés par un contrat qui, tout en mettant en place le mode de leur collaboration, vise à maintenir l'unicité et la rareté de l'œuvre acquise: il prescrit que les deux exemplaires ne peuvent êtres exposés en même temps, que l'exemplaire propriété de Marie Glisser-Broodthaers doit être exposé dans d'autres lieux que le MNAM et que le cartel doit informer les visiteurs de son identitét ${ }^{15}$.

Voici un dernier cas de fabrication d'un deuxième exemplaire d'une œuvre originale qui témoigne aussi de l'action des œuvres contemporaines et de leurs auteurs sur les pratiques de l'institution muséale. Il s'agit de la production du deuxième exemplaire de Moon Is the Oldest TV de Nam June Paik, réalisé par l'artiste dans le cadre de la rétrospective organisée par le Guggenheim Museum en 2000 intitulée The Worlds of Nam June Paik (ill. 3). Cette œuvre a été 
acquise par le MNAM en 1985. Elle été inscrite à son inventaire "sous la forme d'une sculpture vidéo en exemplaire unique ${ }^{16}$. Elle est constituée de téléviseurs posés sur des socles dont le nombre varie. Paik a déposé, sur leur tube cathodique, un aimant qui interfère avec le signal électronique et transforme le point en cercle, demi-cercle et fragment, produisant ainsi une représentation des différentes phases du cycle lunaire'7.

En 1999, le MNAM a agréé à la demande du Guggenheim Museum et de Nam June Paik de fabriquer une copie d'exposition qui soit adaptée à l'espace de ce musée. Nathalie Leleu note qu'une simple autorisation écrite a traversé l'Atlantique ${ }^{18}$. Le MNAM a, par ailleurs, exigé que cet exemplaire soit réalisé exclusivement pour la rétrospective et que le cartel précise qu'elle est une copie d'exposition d'une œuvre originale, propriété exclusive du Centre Pompidou et đuu Musée national d'art moderne. De plus, il a demandé que sa copie Beta et son vidéodisque lui soient remis au terme de l'exposition, ainsi qu'une documentation photographique de l'installation' ${ }^{19}$.

Or, l'œuvre exposée au Guggenheim Museum n'est pas identique à l'original, bien qu'elle établisse un rapport de complémentarité avec celui-ci. Son cartel la désigne comme une version colorisée réalisée en 2000 de l'œuvre originale de $1965^{20}$. Les variations concernent le nombre de moniteurs, les images, leur procédé technologique ainsi que le mode d'occupation de l'espace par l'œuvre. L'œuvre était constituée de treize moniteurs couleur de 13,25 pouces alors que l'œuvre de la collection du MNAM (Paris) est composée de téléviseurs de 20 pouces, noir et blanc et couleur, dont le nombre peut toutefois varier. Paik a aussi opéré une migration technologique en ayant recours à la saisie d'images sur bande vidéo. Il a également ajouté une bande vidéo montrant un vol d'oiseau en image de synthèse tirée de Full Moon (1977); il a légèrement colorisé ou bleuté l'ensemble des bandes vidéo ${ }^{21}$. Enfin, il a modifié le dispositif de mise en vue de l'œuvre qui avait été présentée à la lumière naturelle dans des demi-salles du musée. De plus, dans le cas du musée
16

LELEU, Nathalie. "L'art vivant, le musée et leurs petites économies. Pièce en 6 cuures et 5 épisodes". L'art même, $n^{0} 17$, Ministère de la Communauté française de Belgique. <http://www2.cfwb.be/lartmeme/ framo03.htm> (consulté en avril 2010).

17

VAN ASSCHE, Christine. "Une histoire de vidéon. Vidéo et après, la collection vidéo du Musée national d'art moderne. Paris: Centre Pompidou, Editions Carré, p.12.

18

LELEU, "L'art vivant, le musée et leurs petites économies...», op. cit. 19

Archives de collection, Musée national d'art moderne (Centre Pompidou). Note à l'attention de $G$. Cerutti de C. Van Assche, 4 mai 2000 20

LELEU, "L'art vivant, le musée et leurs petites économies...n, op. cit. <http://www2.cfwb.be/lartmeme/framoo3.htm> (consulté en septembre 2010). Elle cite le cartel: "7965, colored version 2000. Collection CNAC-GP, MNAM, Paris. This variation of 65 original created for The worlds of Nam June Paik is made possible by the NASA Art program."

21

Description de l'installation de l'œuvre par John Hanhardt, conservateur au Guggenheinm Museum et commissaire de l'exposition. Courriel de John Hanhardt à Justine Lebeau, assistante de recherche du projet "Réexposition et pérennité de l'art contemporain", sous la direction de Francine Couture, Département d'histoire de l'art, UQAM, 24 novembre 2008 . 






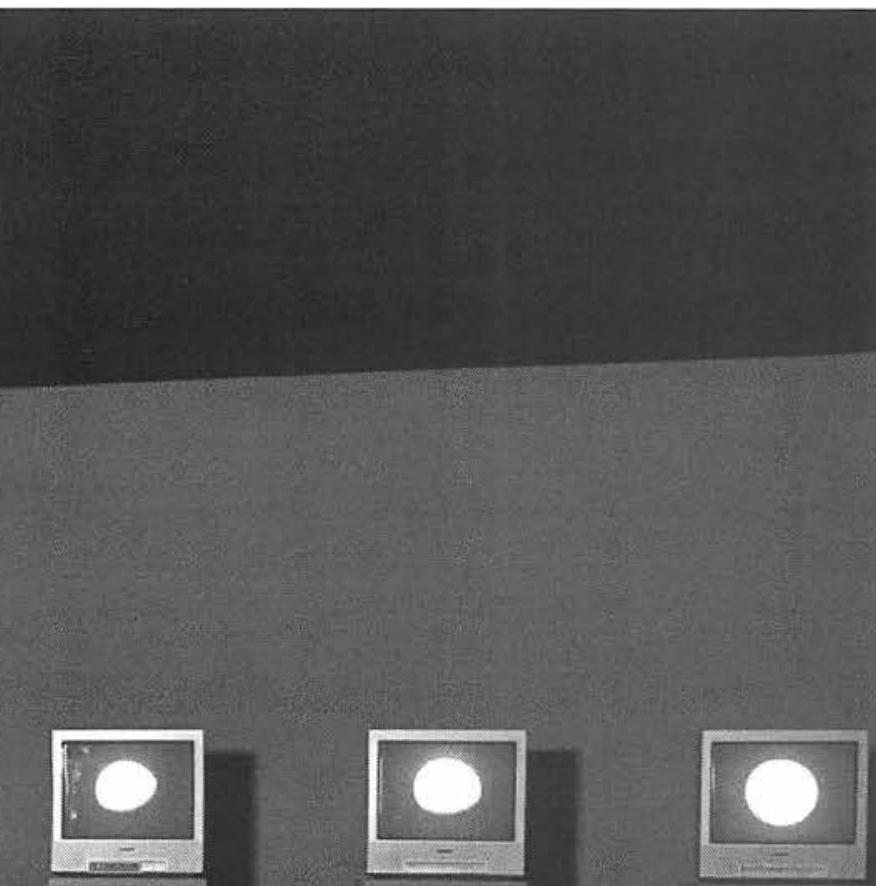

\section{Illustration 3}

Paik, Nam June (1932-2006), ↔ Moon Is the Oldest TV, 1965-1992. Installation vidéo, AM1985-142. Collection Musée national d'art moderne, Centre Pompidou, Paris, France. Crédit photo: CNAC/MNAM/Dist. Réunion des Musées nationaux / Art Resource, NY 
22

Archives de collection, Musée national d'art moderne (Centre Pompidou). Document sans titre produit par le Musée national d'art Moderne (Centre Pompidou, Paris) lors de l'acquisition de l'ceuvre: il est mentionné que les postes de télévision sont installés dans une salle obscure. Voir aussi " Descriptif minimum d'une œuvre", 23 novembre 1994 : il est dit que l'œuvre est présentée dans un espace obscur. Des fiches identiques ont été produites le 6 mai 1992 et le 10 juillet 1995; "Fiche technique de prêt», non datée: elle prescrit que la salle doit être entièrement noire; "Nam June Paik Moon Is the Oldest TV, 1965-1992 ", Collection Nouveau Média, Installation. Paris: Centre Pompidou, 2006, p. 230-231. 23

Archives de collection, Musée national d'art moderne (Centre Pompidou). Note de Christine Van Assche à l'attention de G. Cerutti, 4 mai 2000.

24

WOOSTER, Ann-Sargent. "Nam June Paik». Art News, vol. 75. mai 1976, p. 128; HANHARDT, John. Nam June Paik. New York: Whitney Museum of American Art, catalogue de l'exposition tenue du 30 avril au 27 juin 1982 , p. 99.

25

VANASSCHE, Christine. Vidéo et après, la collection vidéo du Musée national d'art moderne. Paris: Centre Pompidou, Editions Carré, 1992 p. $46 \mathrm{r}$.
Guggenheim de Bilbao, où l'œuvre a aussi été exposée, les moniteurs ont été disposés très haut sur un mur. Ces modes de mise en vue sont différents de celui fixé par le MNAM qui a toujours installé l'œuvre dans une salle obscure; ce type de disposition avait été désigné par l'institution comme un des éléments constitutifs de l'œuvre ${ }^{22}$. Toutes ces variations ont été relevées par la conservatrice des arts médiatiques du MNAM qui a reproché au musée Guggenheim de ne pas avoir respecté les dispositifs originaux confirmés par les présentations antérieures de l'œuvre. Elle mentionne toutefois que le musée n'est pas seul responsable de ces modifications, car elles ont été faites avec l'accord de Nam June Paik, et sous sa responsabilité23.

S'engage alors à l'intérieur de l'institution parisienne une discussion à teneur juridique visant à définir le statut de ce deuxième exemplaire et à évaluer comment elle peut préserver la valeur d'unicité de l'œuvre acquise.

Avant d'examiner plus attentivement cette discussion, mentionnons que Moon Is the Oldest TV a été originellement conçue comme étant variable et évolutive. Au moment de sa mise en vue inaugurale en 1965, à la galerie Bonina à New York, elle était constituée de douze téléviseurs noir et blanc. En 1976, lors de sa présentation à la galerie René Block (New York), Paik les a remplacés par des téléviseurs couleur tout en maintenant le procédé technique originel de production des images. Cette nouvelle occurrence a donc donné lieu à la production d'une nouvelle version, l'œuvre ayant été datée 1965-197624. Paik a cependant continué de réactualiser la première version à maintes reprises. Par ailleurs, lors l'acquisition de Moon Is the Oldest TV par le MNAM en 1985, il a ajouté quatre moniteurs couleur. Pour signaler ces différents états de l'œuvre, sa fiche descriptive mentionne qu'elle a été conçue en 1965, reprise en 1976 et perfectionnée en 1985 , date de son acquisition par le musée ${ }^{25}$.

Par ailleurs, cette œuvre enfreint la convention muséale de sauvegarde de l'intégrité matérielle originelle de l'œuvre d'art par le fait que ses éléments proviennent de l'uni- 
vers industriel. C'est l'avis de Nathalie Leleu qui souligne que Moon Is the Oldest TV est constituée d'objets manufacturés qui sont interchangeables et qui ont été remplacés. Ainsi, parmi les téléviseurs qui la composent, certains ont été acquis par Paik en 1960 à New York et d'autres auprès de brocanteurs parisiens à la fin des années $1980^{26}$. De plus, son insertion dans l'univers technologique s'est confirmée, en 1994, lors de la captation vidéo par l'artiste des effets de lumière représentant les phases du cycle lunaire. Bien que cette saisie ait été réalisée pour des raisons de préserver la pérennité de l'œuvre et que les bandes vidéo aient été qualifiées par le musée de copie de survie, "la vidéo incorporée à cette œuvre plastique, souligne Nathalie Leleu, l'introduit dans la catégorie des arts reproductibles qui échappent aux critères d'unicité et de l'exemplaire original " ${ }^{27}$, ce qui constitue une condition favorable à l'édition de l'œuvre en plusieurs exemplaires. La version migrée a été, en effet, présentée dans des expositions ${ }^{28}$, bien que la fiche de l'œuvre publiée dans le récent catalogue de la collection des nouveaux médias indique que son aspect performatif continue d'être réactualisé ${ }^{29}$.

Ce caractère variable et évolutif de Moon Is the Oldest TV a été documenté par le MNAM qui a consigné ses états successifs. Dans une "Lettre ouverte à Nam June Paik", la conservatrice des arts médiatiques aborde, dans ces termes, ce caractère spécifique de l'œuvre: "Cette œuvre semble être dans ton corpus celle qui demeure ouverte, celle que tu ne désires pas achever, celle que tu te plais à transformer à chaque présentation, celle qui n'a pas trouvé de repos, et même son statut définitif ${ }^{30}$. " Le MNAM n'avait toutefois pas prévu que les variations de l'œuvre résultant de sa réexposition généreraient la production d'une nouvelle œuvre. La direction juridique de l'institution s'est référée à la jurisprudence pour affirmer que l'œuvre présentée au Guggenheim Museum constitue une nouvelle œuvre originale, et non pas une copie d'exposition, car l'artiste a apporté de nombreuses modifications, dont l'utilisation de nouveaux moniteurs et l'introduction d'images numériques. Le MNAM ne détient ni droit de propriété, ni droit d'exploitation sur cette version adaptée d'une œuvre de sa collec-
26

LELEU, Nathalie. L'art d'accommoder les restes, <http://www.vacarme. eu.org/article1573.html) (consulté en avril 2010).

27

Id.

28

Les descriptifs de l'œuvre datant respectivement de 1992, 1993 et 1994. produits par le Musée national d'art moderne, mentionnent, parmi les éléments constitutifs de l'œuvre, onze bandes vidéo et les lecteurs DVD Pal en boucle. C'est ce qu'indiquent aussi les fiches de prêt qui prescrivent aux institutions de se procurer des lecteurs DVD. (Archives de collection, Musée national d'art moderne, Centre Pompidou. Fiche de prêt MNAM/CCl, Centre Pompidou, Moon is the Oldest TV, 1965-1992, n.d.)

29

"Nam June Paik Moon Is the Oldest TV, 1965-1992". Collection Nouveau Média Installation, Paris: Centre Pompidou, 2006, p. 230-231.

30

BUBMANN, Klaus et Florian MATZNER. Nam June Paik eine DATA base. Deutscher Pavillon, Stutgart: La Biennale di Venezia XLV Espozione Internatiotale d'Arte, 13 juin - 10 octobre 1993, Cantz éditeur, 1993. p. 147 
31

Archives de collection, Musée national d'art moderne Centre Pompidou. Note de Cabriel Ballif, attaché juridique de la direction juridique et financière de Pompidou à Christian Van Assche, 18 décembre 2001. tion; celle-ci doit donc être considérée comme une création nouvelle et originale appartenant à l'artiste ou au Cuggenheim Museum, producteur de l'exposition ${ }^{3}$. Il existe dorénavant deux œuvres portant le titre Moon Is the Oldest $T V$, celle de la collection du Musée national d'art moderne datée 1965, 1976, 1985 et la version colorisée présentée au Cuggenheim Museum et conservée au Nam June Paik Art Center à Séoul. Cette situation a eu toutefois une répercussion sur les pratiques d'acquisition d'une installation vidéo par le MNAM. L'institution a apporté des modifications à son contrat d'acquisition visant principalement à préserver la valeur de rareté de l'œuvre acquise et à restreindre l'exercice du droit moral de l'artiste ; il est ainsi prescrit que l'artiste et ses ayants droit ne pourront en aucun cas ni reproduire ses éléments, dont en particulier les bandes vidéo, ni reproduire à l'identique un exemplaire supplémentaire de l'œuvre acquise sous quelle que forme que ce soit.

\section{Qu'est-ce qu'une œuvre intégrale?}

Nous abordons maintenant le deuxième cas de figure annoncé précédemment qui consiste en la présentation d'une œuvre déjà produite sur le mode de un ou de plusieurs de ses éléments constitutifs. Ce mode d'exposition interroge la représentation de cette œuvre comme un ensemble unique doté d'une identité spécifique et questionne les modalités de reconnaissance de son intégralité matérielle et de son intégrité conceptuelle. Pour illustrer ce cas de figure, nous analyserons deux exemples résultant d'une association étroite entre leurs conditions d'acquisition et d'exposition: Léviathan Thot d'Ernesto Neto dont une partie a été acquise, comme œuvre autonome, par le Fonds national d'art contemporain (France) à la suite de son exposition intégrale au Panthéon, à Paris, en 2006 ; et Les Piques d'Annette Messager, une œuvre évolutive et sérielle dont l'artiste a produit plusieurs exemplaires qu'elle a réunis pour constituer un grand ensemble lors de sa rétrospective Les Messagers tenue au Centre Pompidou en 2007.

À l'automne 2006, dans le cadre du Festival d'automne à Paris, Ernesto Neto exposa Léviathan Thot qui a été comman- 
dée à l'artiste par le ministère de la Culture et des Communications de France et le Festival d'Automne (ill. 4). Le public, qui a fait l'expérience de sa mise en vue inaugurale, a vu une installation monumentale représentant le corps du Léviathan, monstre du livre de Job, composée de cinq parties faites de tulle de lycra qui étaient suspendues dans l'espace. L'artiste semble les avoir considérées comme pouvant exister de façon autonome puisqu'il leur a donné un titre se référant aux parties du corps du monstre représenté : Léviathan tête toth, Léviathan doigt toth, Léviathan corps toth, Léviathan toth toth et Léviathan main toth. Cette dernière partie a été acquise par le Fonds national d'art contemporain (France) qui avait été responsable de la mise en vue inaugurale de l'œuvre intégrale. Par une telle acquisition cette institution, avec l'accord de l'artiste, dote Léviathan main toth d'une identité spécifique et numérique et propose ainsi que celle du Léviathan Thot résulte, aurait pu écrire Gérard Genette à propos de cette œuvre, d'u une relation intentionnelle d'assemblage d'éléments autonomes" ${ }^{32}$. Le théoricien donne en exemple de ce mode d'immanence d'une cuvre d'art le volet d'un polyptique car, considère-til, il constitue un "élément dans un groupe", mais il peut aussi être présenté comme une œuvre autonome. Ernesto Neto et le Fonds national d'art contemporain ont réactualisé ce double mode de manifestation. La mise en vue inaugurale de Léviathan Thot a été minutieusement documentée par l'institution collectionneuse. Et, à la demande de l'artiste, elle s'est engagée à prêter Léviathan main toth lors d'une exposition qui présenterait le Léviathan Thot dans son intégralité33 . Ce protocole alloue donc à Léviathan main toth un double statut générant deux modes d'immanence, celui de l'œuvre autonome et celui de partie d'un ensemble.

L'œuvre évolutive et sérielle d'Annette Messager, Les Piques, met en place un autre mode de conjugaison de la relation entre les parties d'une œuvre et sa représentation comme un ensemble (ill. 5). A la différence de Neto, l'artiste a conçu Les Piques comme devant être variable, car, lors de ses expositions, elle a modifié le nombre de ses éléments constitutifs. Ainsi, à l'occasion de sa présentation à la Josh Baer Gallery à New York, en 1993, l'œuvre était constituée
32

CENETTE, op.cit., p. 242

33

Courriel d'Aude Bodet, conservatrice, Fonds national d'art contemporain (France), à Francine Couture, 22 janvier 2007 . 


\section{Illustration 4}

Neto, Ernesto (1964--), Léviathan Thot, textile, polystyrène, sable, 2006, Place du Panthéon, Paris, DR CNAP. Crédit photo: Y. Chenot, Paris 


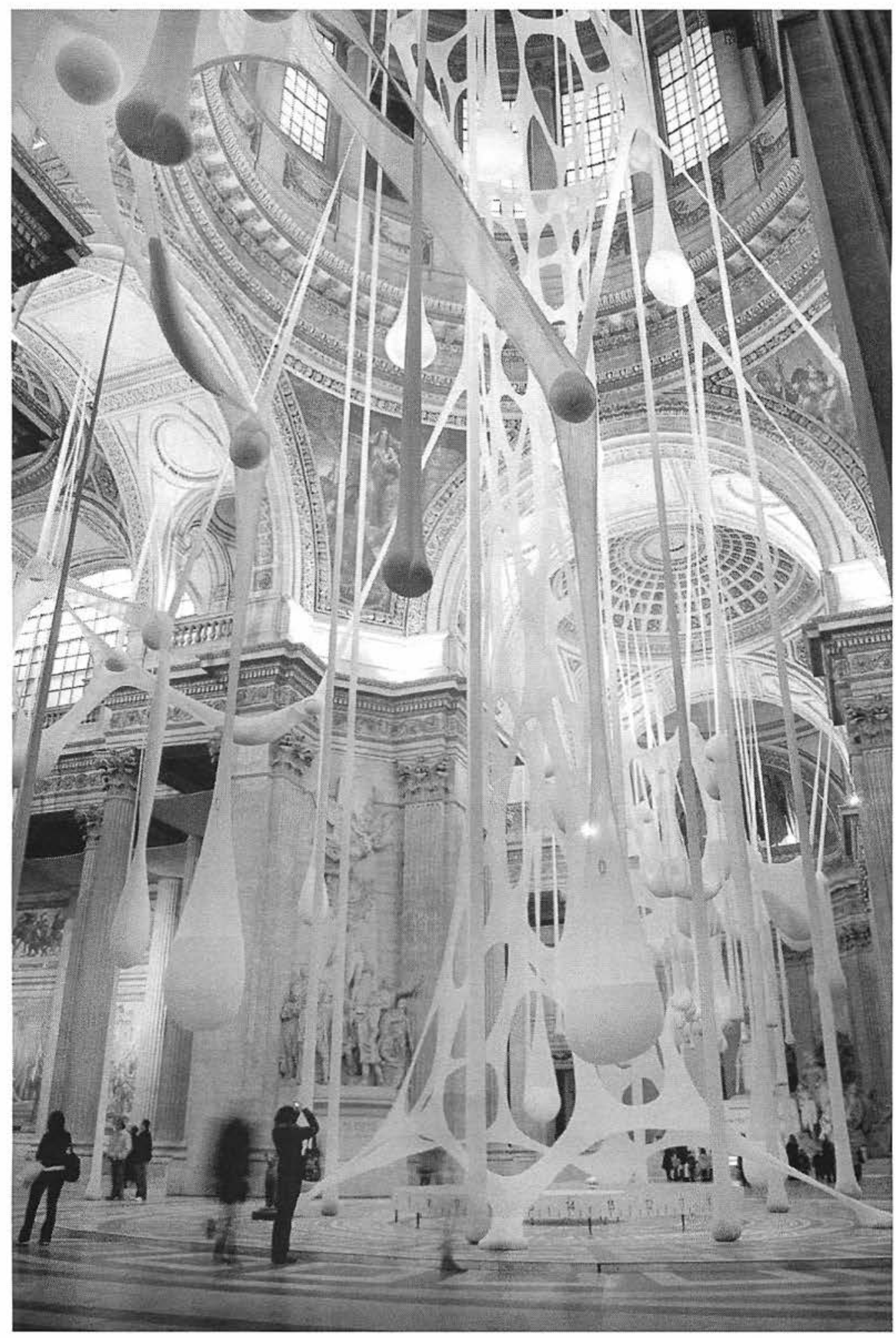









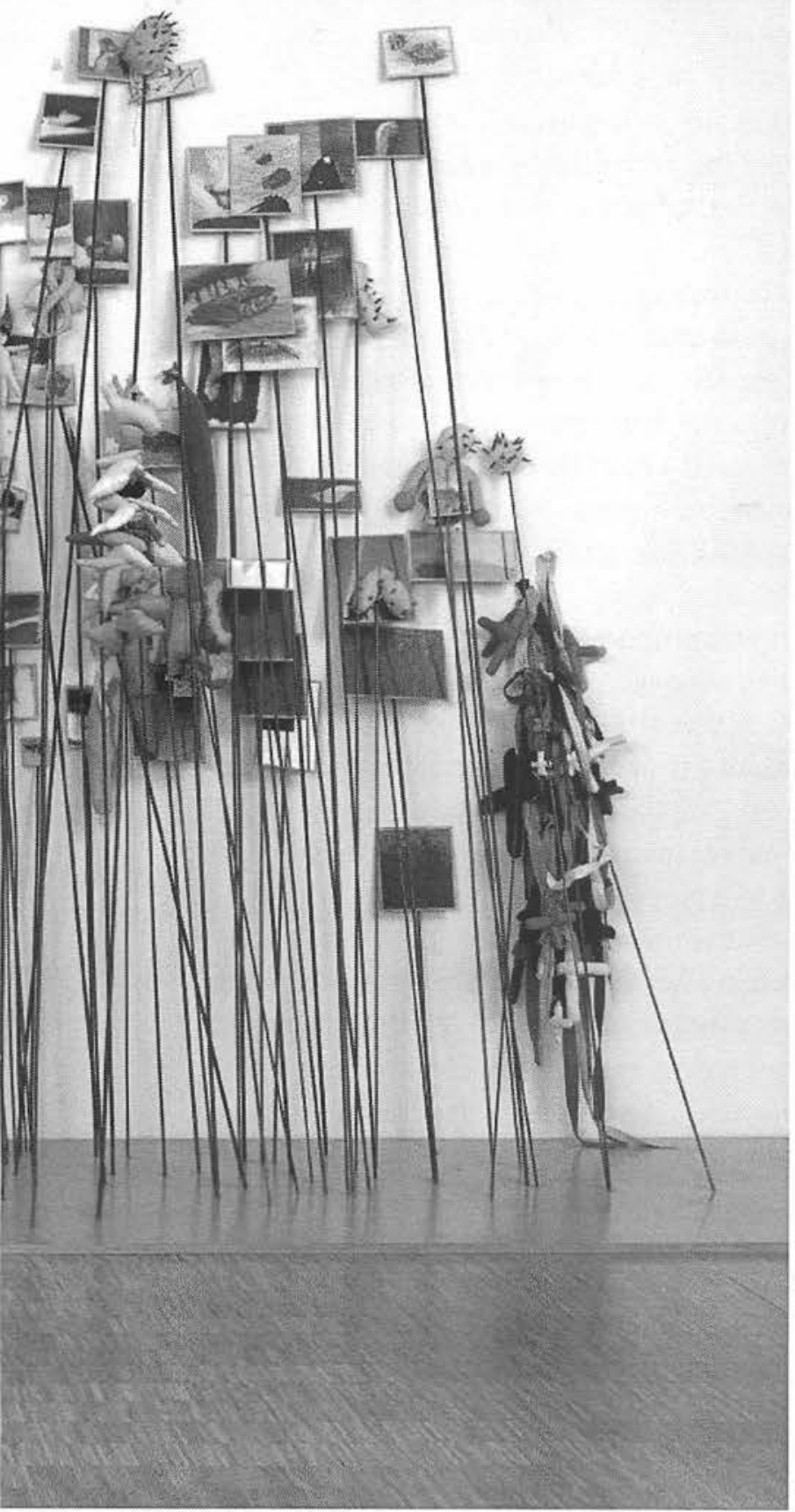

mlustration 5

Messager, Annette (1943--), $\odot$ ARS, NY, Les Piques 1992-93, médias mixtes, $250 \times 800 \times 425 \mathrm{~cm}$. AM1994-85. Photo: Philippe Migeat. Collection Musée national d'art moderne, Centre Pompidou, Paris, France. Crédit photo: CNAC/MNAM/Dist. Réunion des Musées nationaux / Art Resource, NY 
34

HACEN, Charles. "Annette Messager ". The New York Times, 11 juin 1993; PERCHUCK, Andrew. "Annette Messager". Artforum, décembre 1993. <http://findarticles.com/p/articles/mi_moz68/ is_n4_v32/ai_14890871/> (consulté en mars 2010).

35

Archives des collections, Musée national d'art moderne (Centre Pompidou). Document non titré concernant l'acquisition de Les Piques. 36

"Les piques' (The Pikes'), a rich and complex sculptural assemblage, is half of an installation first shown at the Pompidou Centre in Paris." (Tate Callery Artists Catalogue File Foreign. Messager Annette born 1943 PC.4.2.1, The Pikes, Les Piques, 1992-1993. T08436 A21255. $59 \mathrm{~b} / 03 / 2 \mathrm{G}$.)

Chantal Crousel, dans un fax envoyé par à Virginia Button en date du 20 novembre 1997, en donne la description suivante: "Les Piques", 1992-1993. Installation consisting of approx. 130 metal poles, bearing pastel drawings on paper under glass, handmade fabric objects, nylon-masks, parts of dolls etc.). Installation flexible, approx. $10 \mathrm{~m}$ long, $2.50 \mathrm{~m}$ high (see detail on cover catalogue LACMA/MOMA and enclosed ektachrome). Exhibited originally at Centre G. Pompidou, L'envers des choses 1993. "(Document Tate Artists' Catalogue File Foreign, op. cit.) de 183 tiges de métal de 100 à 250 centimètres de longueur, à l'extrémité desquelles étaient empalées des formes faites de tissus représentant des fragments de poupées auxquels étaient associés des dessins scellés sous verre réalisés au crayon ou à la plume. Ces tiges renvoient au régime de terreur de la Révolution française et à l'usage de piques où étaient empalées les têtes des aristocrates qui avaient été guillotinés. Appuyées sur deux murs contigus, elles occupent 50 pieds de l'espace de la galerie ${ }^{34}$. Lors de la troisième exposition de l'œuvre, intitulée L'envers des choses: Annette Messager, Cindy Sherman, Ceorge Kuchar, au Studio des galeries contemporaines du Musée national d'art moderne à Paris (7 septembre - 11 octobre 1993), l'artiste a réduit le nombre de piques de 183 à 130 tout en réactualisant le dispositif de mise en vue de l'exposition de New York, les tiges de métal ayant été appuyées sur deux murs situés en angle, alors qu'à l'occasion de l'exposition Big Bang en 2005, au Musée national d'art moderne (Centre Pompidou), pour la première fois, l'œuvre se déployait sur un seul mur.

Par ailleurs, tout en ayant doté Les Piques d'un potentiel de variabilité, Annette Messager l'a également conçue comme se développant dans une série et elle en a produit plusieurs exemplaires qui ont été acquis par des collectionneurs, dont le MNAM et la Tate Gallery. Bien que ces exemplaires soient reconnus par ces institutions comme des œuvres originales dotées de leur propre identité numérique et spécifique, ils portent tous le même titre, indiquant ainsi qu'ils ont été pensés comme des éléments d'une série procédant d'un même projet conceptuel. En 1994, le MNAM a acquis un exemplaire qui n'était pas identique aux versions précédemment présentées. Le musée avait offert à l'artiste d'acheter " un ensemble suffisamment représentatif de cette œuvre" "35, composé par Annette Messager. La légende de l'œuvre indique qu'il est formé de 125 piques d'acier, 65 dessins au crayon et au pastel sous verre, objets, tissu, bas de nylon, ficelle, morceaux de peluches, crayons de couleur, et que ses dimensions sont variables. En 1998, la Tate Gallery a acheté l'exemplaire qui a été exposé au Centre Pompidou en 1993 dans l'exposition L'envers des choses ${ }^{36}$. Le rapport de la visite d'atelier d'Annette Messager par la 
conservatrice Virginia Button de la Tate Gallery procure de l'information précise sur l'état de l'œuvre acquise, indiquant qu'elle n'est pas identique à l'exemplaire du Musée national d'art moderne ${ }^{37}$. On y lit que l'œuvre est composée de 140 tiges de métal, de 76 dessins faits au crayon ou à la plume de couleur, placés sous verre. Par ailleurs, sa description spécifie que, sur le plan conceptuel et celui de ses composantes iconographiques, elle s'inscrit bien dans la série Les Piques. Le relevé des consignes données par l'artiste concernant le mode de disposition de l'œuvre mentionne que ce sont celles de sa mise en vue inaugurale. La conservatrice a également retenu qu'Annette Messager a prescrit le maintien du caractère variable de l'œuvre par l'adaptation de ses dimensions à l'espace d'exposition. Elle a en outre mentionné qu'elle n'adhère pas au principe muséal de la préservation de son état matériel originel et qu'elle a recommandé le remplacement de ses composantes de tissus, jugeant que leur caractère fait main est conciliable avec leur détérioration.

Quelques années après l'acquisition des exemplaires Les Piques par le MNAM (Paris) et la Tate Gallery (Londres), Annette Messager a réaffirmé leur caractère variable en manipulant leur statut d'œuvre autonome ou en les traitant, pour le temps d'une exposition, comme éléments d'un ensemble. En effet, lors de l'exposition Les Messagers, tenue au Centre Pompidou, du 6 juin au 17 septembre 2007, elle les a réunis, sous le titre originel Les Piques, afin de constituer un grand ensemble disposé selon le dispositif de la mise en vue inaugurale. Toutefois, bien que cet ensemble ait été offert au regard des spectateurs comme une seule œuvre, la notice du catalogue a signalé l'identité numérique de chacun des exemplaires qui la constitue par la mention de leur date de production, leurs caractéristiques matérielles et leur insertion respective dans les collections de la Tate Callery et du Musée national d'art moderne. Annette Messager avait déjà réalisé un tel regroupement lors de l'exposition Annette Messager, une rétrospective organisée par le Los Angeles County Museum of Art qui réunissait dans un même ensemble trois exemplaires ${ }^{38}$; celui du MNAM, celui des collections Michael Harris Spector et du
37

Document Tate Artists' Catalogue File Foreign (op. cit.). Report on Annette Messager's Les Piques (following a studio visit by Virginia Button, December 1997). 38

Rétrospective organisée par le Los Angeles County Museum of Art (15 juin au 3 septembre 1995), le Modern Art Museum, New York (12 octobre au 16 janvier 1996), et I'Art Institute of Chicago ( 17 février au 5 mai). 
39

Courriel de Carol S. Eliel, curator Modern and Contemporary Art, Los Angeles County Museum of Art, à Justine Lebeau, assistante de recherche du projet " Réexposition et pérennité de l'art contemporainn, sous la direction de Francine Couture, Département d'histoire de l'art, UQAM. 30 octobre 2009. 40

Courriel envoyé par Siobhan McCracken, assistant curator, Hayward Gallery, à Justine Lebeau, 8 juillet 2009 .
Dr Joan Spector, et celui de l'artiste ${ }^{39}$. Ce mode d'immanence de Les Piques n'a cependant pas été réitéré à chacune des présentations de l'exposition Les Messagers qu'ont reçue plusieurs musées. Ainsi, au Espoo Museum of Modern Art (EMMA) (Finlande), ce n'est que l'exemplaire du Musée national d'art moderne qui a été mis en vue. L'œuvre a été installée sous la supervision de l'artiste en collaboration avec des techniciens et des conservateurs du Centre Pompidou et du musée finlandais; ils ont réactualisé son mode de mise en vue inaugurale. A la Hayward Gallery, c'est l'exemplaire de la Tate Gallery qui a été exposé ; l'œuvre fut installée par Annette Messager en collaboration avec le restaurateur de cette institution ${ }^{40}$. Dans le contexte d'une collaboration étroite entre l'artiste et l'institution muséale, l'exposition Les Messagers a donc donné lieu à la manipulation artistique du statut des exemplaires Les Piques en les présentant sur les modes de l'œuvre autonome et d'une partie d'un ensemble ; cette opération, toutefois, n'a pas porté atteinte à leur identité numérique ou à leur valeur d'unicité préservée par le musée.

\section{La réactualisation d'une œuvre allographique et la multiplication des exemplaires}

Abordons maintenant le troisième cas de figure, celui de la réactualisation d'une œuvre allographique. À la différence des exemples précédemment analysés, chacune de ses expositions donne lieu à la production d'un exemplaire qui n'est cependant pas doté d'une identité numérique distincte, car il résulte de l'actualisation d'une notation ou d'un script qui, par la description des composantes de l'œuvre et des conditions de sa prestation, a établi son identité spécifique et son caractère unique.

L'œuvre de Claude Rutault Toiles à l'unité, 1973 / Légendes, 1985 acquise en 1988 par le MNAM est exemplaire de cette catégorie d'œuvres d'art (ill. 6). Elle a été acquise sur le mode d'une notation constituée des définitions-méthodes identifiées par les numéros 1 et 145 qui ont été réunies par l'artiste. Elles s'énoncent ainsi : 
1. TOILES À L'UNITÉ, 1973

définition-méthode

une toile tendue sur châssis peinte de la même couleur que le mur sur lequel elle est accrochée, sont utilisables tous les formats standards disponibles dans le commerce qu'ils soient rectangulaires, carrés, ronds ou ovales. L'accrochage est traditionnel ${ }^{47}$.

145. LÉCENDES, 1985

définition-méthode

principe : une grande toile peinte de la même couleur que le mur sur lequel elle est accrochée. juste à sa droite, sans que la place soit mécaniquement définie mais là où figure habituellement le cartel, une très petite toile no. o paysage ou marine ( $12 \times 18 \mathrm{~cm}$ ou $10 \times 18 \mathrm{~cm}$ ) est accrochée, également peinte de la même couleur que le mur. s'il s'agit d'un mur non peint, la légende n'est pas peinte non plus.

il est possible de légender un mur vide, l'œuvre d'un autre artiste, une sculpture et une photographie ou une autre $\mathrm{d} / \mathrm{m}$. il est également possible d'associer la $\mathrm{d} / \mathrm{m} 34$ : un papier peut être légendé par une toile et un papier peut légender une toile

le nombre de réalisations n'est pas limité42.

Lors de l'acquisition de cette œuvre, l'artiste a également transmis au musée un descriptif qui agit aussi comme script par la description des éléments constitutifs de la matérialisation de l'œuvre acquise conformément aux principes des définitions-méthodes 1 et 145. Est joint un diagramme produit par l'artiste montrant différentes installations possibles. Ce script ou ce récit autorisé de Rutault agit également comme certificat d'authenticité, car il prescrit les conditions de l'authentification des actualisations de l'œuvre en déclarant qu'elles doivent être aussi suivies par un descriptif ${ }^{43}$.

Depuis 1989 , le MNAM a produit et documenté six itérations de Toiles à l'unité, 1973 / Légendes, 1985 qui sont toutes composées des éléments fixés par le descriptif de 1988 , mais qui actualisent aussi son caractère variable. La couleur des tableaux et du mur où ils sont disposés est laissée au choix du collectionneur. Il y a eu les versions rose, blanche, beige, verte et jaune ${ }^{44}$. A chacune de ces expositions
41

RUTAULT, Claude. Définition/

Méthodes Le Livre. Paris: Production

Flammarion 4, et CCCTours, 2000 ,

p. 877.

42

Id., p. 1190.

43

Archives de collection Musêe national d'art moderne (Centre Pompidou). Descriptif de l'œuvre signé par Rutault, en 1988. 


\section{Illustration 6}

Rutault, Claude (1941--), @ Toiles à l'unité, 1973 / Légendes, 1985, 6 toiles standards sur châssis ; 3 rectangulaires, 1 carrée, 1 ronde. Collection Musée national d'art moderne,

Centre Pompidou, Paris, France. Crédit photo: CNAC/MNAM/Dist. Réunion des Musées nationaux / Art Resource, NY. Référence ART 404370

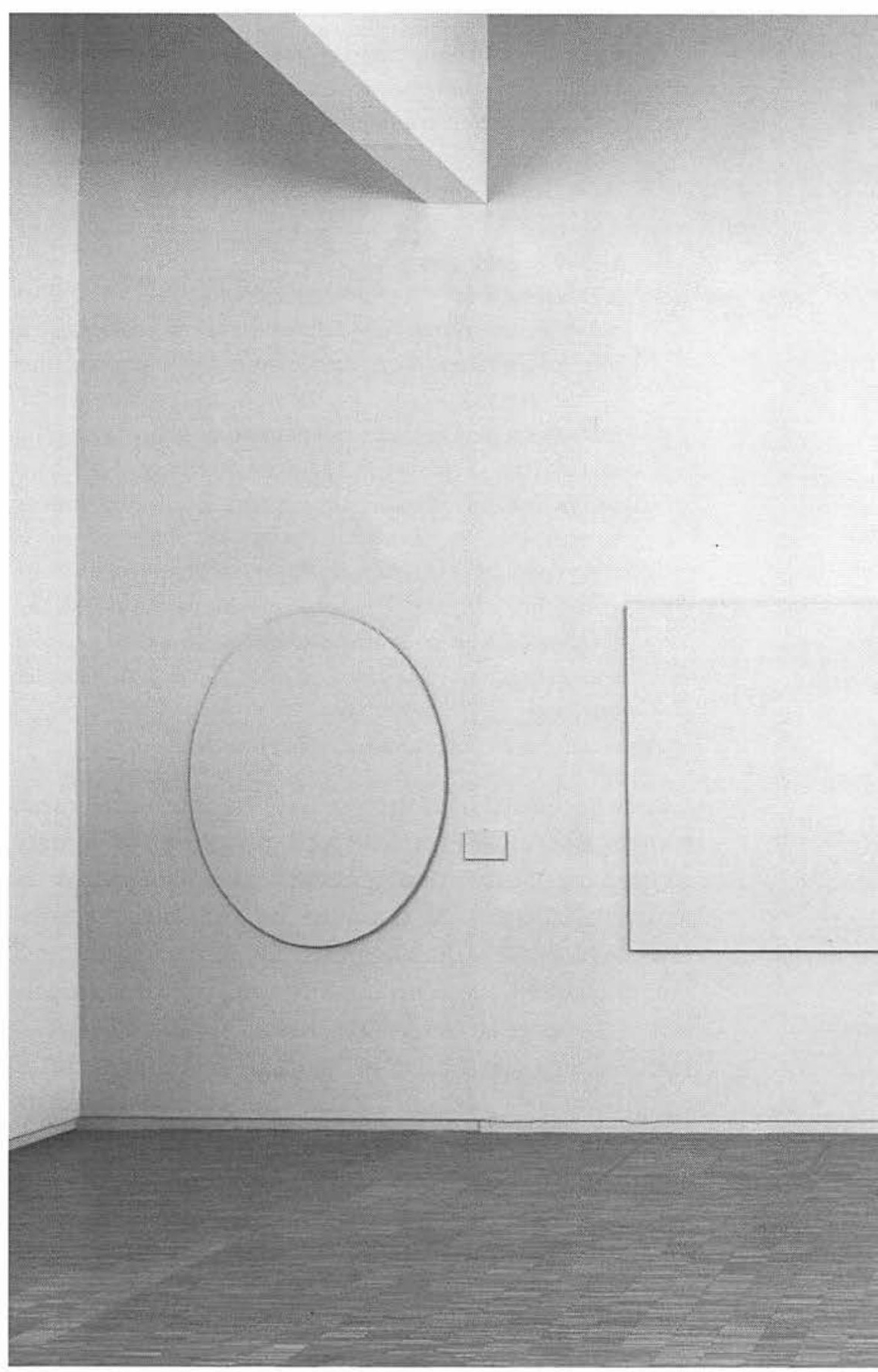



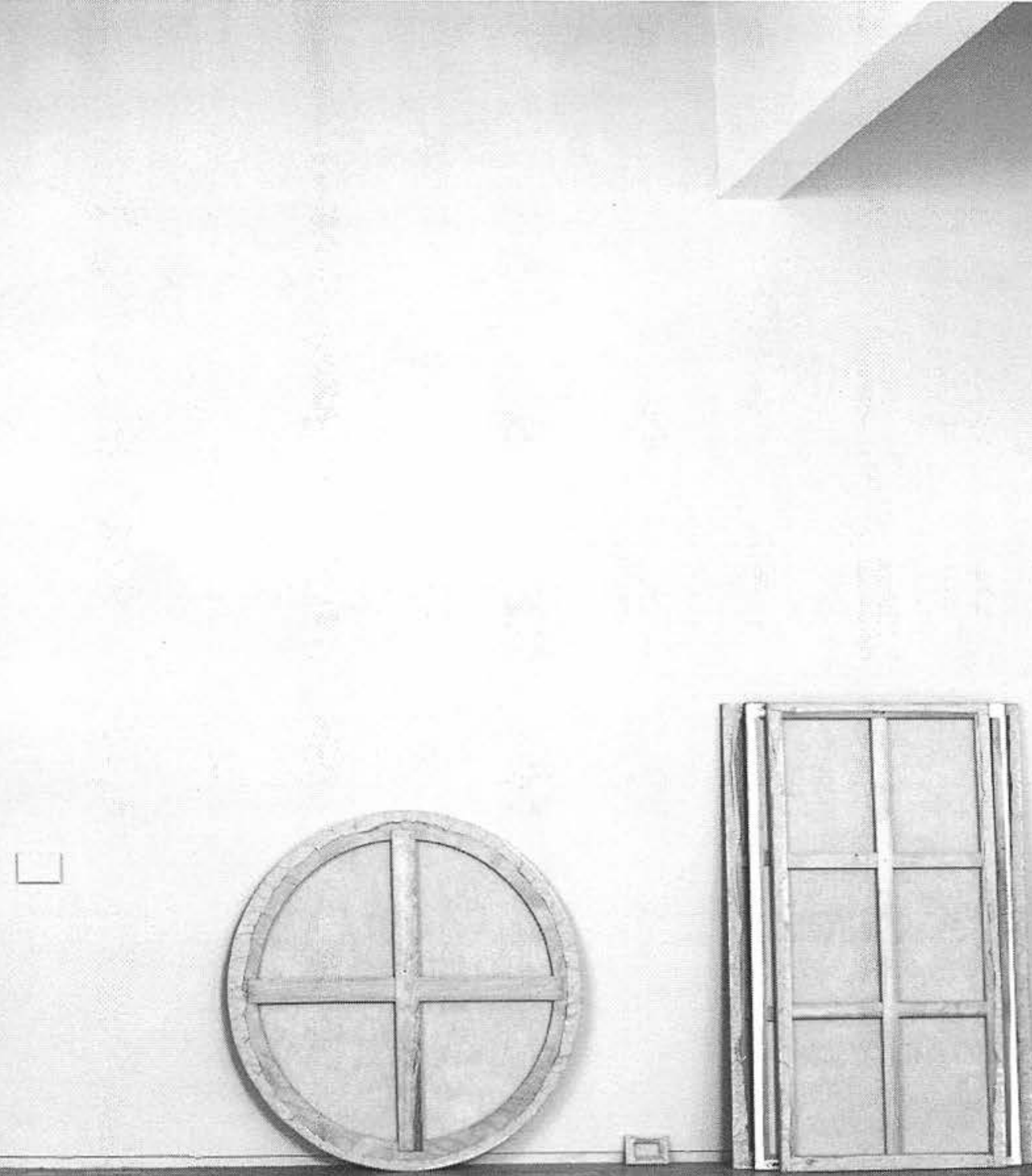


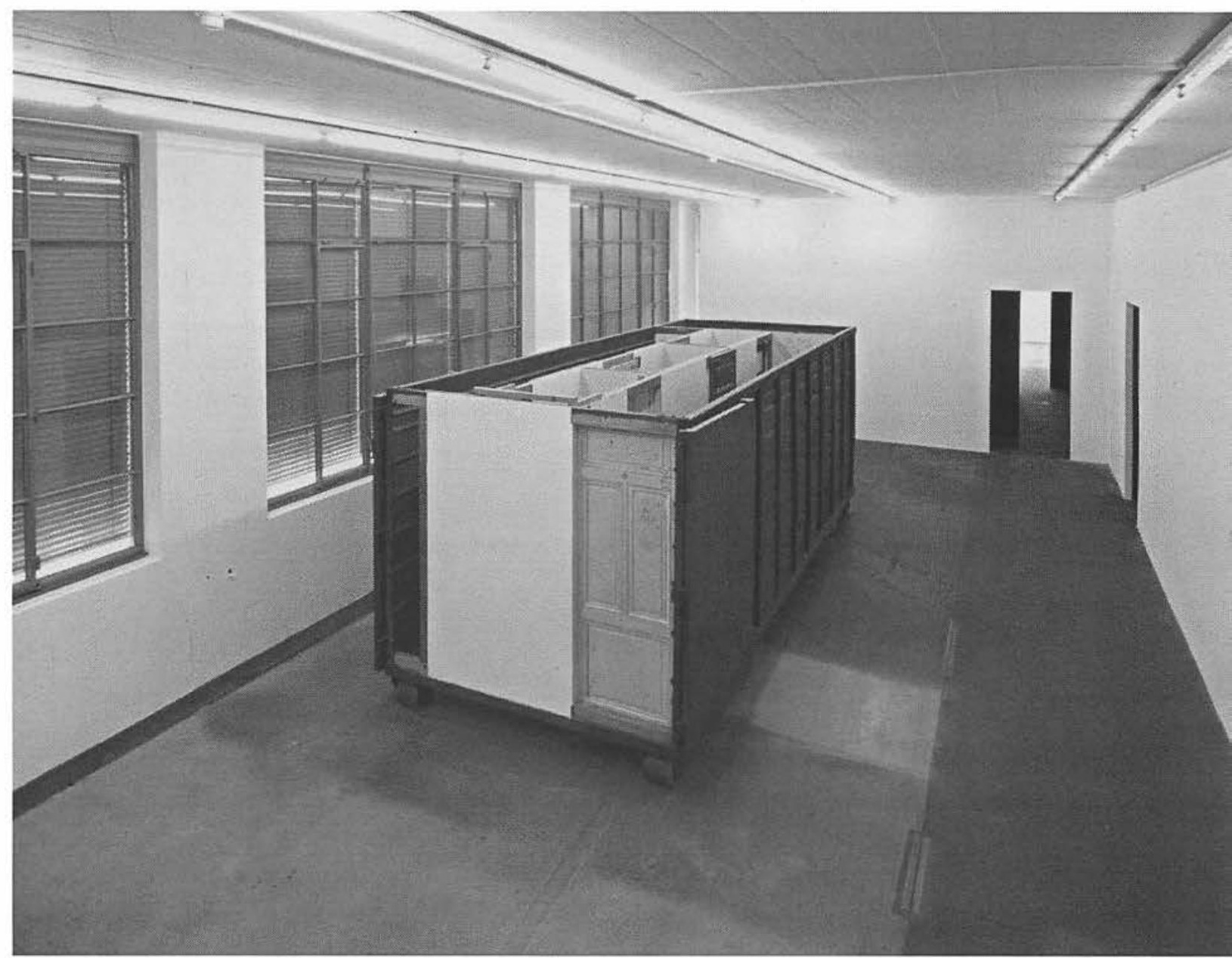

\section{Illustration 7}

Matta-Clark, Cordon (1943-1978),

Open House, Dumpster Duplex, Drag-on, 1972-1985, benne industrielle, cloisons, portes, $248 \times 242 \times 611 \mathrm{~cm}$. Collection Musée d'art moderne et contemporain Mamco, Genève, acquis avec le soutien de donateurs anonymes, de l'Amamco et de Daniel Varenne. Crédit photo: I. Kalkkinen, Mamco 
les tableaux ont été repeints de la même couleur que le mur par les techniciens du musée, l'artiste n'étant pas présent lors de leur fabrication ${ }^{45}$. Le caractère variable de cette œuvre se manifeste aussi par le nombre de toiles composant l'installation ainsi que par leur disposition différente: les versions rose, blanche et verte occupaient trois murs et les versions beige et jaune, un seul mur.

Une fois l'exposition terminée, conformément à la prescription de Rutault, le MNAM a mis en œuvre une procédure de l'authentification de ces actualisations par la production d'un descriptif soumis à la signature de l'artiste et à celle du directeur de l'institution et auquel ont été joints une photographie de l'installation ainsi qu'un échantillon de toile montrant la couleur choisie ${ }^{46}$. Á titre d'exemple, mentionnons le descriptif de l'actualisation de l'œuvre lors de l'exposition Big Bang destruction et création dans l'art du zoe siècle, tenue au MNAM en 2005. Il procède à une description détaillée de l'installation de l'œuvre. Le document donne aussi de l'information sur la marque de peinture utilisée et son numéro de catalogue. Il agit également comme certificat d'authenticité en statuant que toute modification du travail entraîne l'établissement d'un nouveau descriptif établi par écrit et que le non-respect d'une des clauses du présent descriptif entraîne l'impossibilité d'attribuer le travail à Claude Rutault; il est prescrit que l'artiste et le musée doivent le conserver. Il est également mentionné qu'il a un caractère épi-opéral, c'est-à-dire qu'il n'est pas un élément constitutif de l'œuvre, qu'il ne peut être exposé à la place ou à côté đu travail, qu'il ne peut être ni publié, ni reproduit. Ce document a été signé le 21 février 2006 par Alfred Pacquement, directeur du MNAM, et Claude Rutault.

Cette œuvre allographique de Rutault est donc représentative des œuvres d'art contemporaines qui, par la médiation de la notation comme mode de leur manifestation, ont opéré un déplacement de la valorisation de leur matérialité originelle vers celle de leur identité conceptuelle; ce qui a eu pour conséquence l'établissement d'un partenariat professionnel entre l'institution muséale et l'artiste visant à établir un cadre de maintenance de ces

\section{4}

La version rose, exposition de la collection d'art contemporain, Musée national d'art moderne, Centre Pompidou, 1989; la version blanche, Deichtorhallen, Hambourg 1990: la version beige, Les péchés capitaux, La paresse. Musée national d'art moderne, Centre Pompidou, 1996; la version verte, exposition de la collection d'art contemporain, Musée national d'art moderne, Centre Pompidou, 2000; la version jaune, Big Bang, destruction et création dans l'art du $20^{\circ}$ siècle, Musée national d'art moderne, Centre Pompidou, 2005.

45

Entretien avec Evelyne Pomey. Service des archives de collection, Musée national d'art moderne, Centre Pompidou, été 2005. 46

Archives de collection, Musée national d'art moderne, Centre Pompidou. Le descriptif de l'actualisation de l'œuvre lors de l'exposition Big Bang (2005) a été produit par Evelyne Pomey. Service des archives de la collection, Musée national d'art moderne, Centre Pompidou, signé parAlfred Pacquement. directeur de l'institution et Claude Rutault le 21 février 2006. 
47

DISERENS, Corinne. Gordon MattaClark, catalogue d'exposition. Valencia: Centre Julio Conzalez, 1993. p. 155. œuvres pour préserver l'authenticité de leur actualisation, qui, elle, peut être illimitée.

Mais comment est maintenue la pérennité des conditions d'immanence de ces œuvres allographiques lorsque l'artiste décède et que son autorité est transmise aux ayants droit? Les réexpositions d'Open House de Gordon MattaClark, récemment acquise par le Musée d'art moderne et contemporain de Genève, constituent un cas exemplaire de cette situation (ill. 7).

Bien que Matta-Clark n'ait pas produit de notation ou de script décrivant ses composantes, le principe de la conception de cette œuvre relève de caractéristiques des arts allographiques puisqu'elle a été conçue pour être contextuelle et non pérenne. Sa mise en vue inaugurale a eu lieu du 19 au 21 mai 1972 sur Greene Street à New York. Gordon Matta-Clark y a présenté une "habitation" construite avec des amis artistes, dont plusieurs faisaient partie du groupe Anarchitecture qui, en réaction contre l'architecture moderne, préconisait la transformation de structures architecturales existantes. Matta-Clark avait transformé l'espace intérieur d'une benne à ordure en le compartimentant par des cloisons, accompagnées de portes, dont les matériaux avaient été prélevés dans un chantier de démolition. Il y avait inséré un élément sonore en plaçant une radio transistor ainsi qu'une cassette d'une durée de six heures et demie, intitulée Voice Truck, réalisée par le poète Ted Greenwald qui avait enregistré les sons produits par la livraison du Village Voice dans la ville de New York. Le jour du vernissage, cette habitation offerte à la circulation des visiteurs avait été animée par des performances, dont celles de Tina Girouard, de Suzanne Harris et de Barbara Dilley ${ }^{47}$ Gordon Matta-Clark répéta l'expérience sur Greene Street du 21 octobre au 10 novembre 1972. La benne à ordure utilisée était de plus grandes dimensions, elle possédait un escalier menant à une plateforme où avait été installé un barbecue. Il y tourna un film documentant son processus de construction, son exposition et son usage par ceux et celles qui y ont passé du temps, montrant ainsi le caractère performatif d'Open House qui n'était pas qu'un objet, mais 
aussi un acte de construction, un espace pour "performer" et un lieu que l'on fréquente. Cette deuxième itération a aussi donné lieu à des performances par les artistes ayant participé à la première occurrence de l'œuvre.

Après la mort de l'artiste, on relève un nombre important de refabrications d'Open House. Bien qu'il n'existe pas de notation descriptive de ses composantes et de ses conditions de présentation rédigée par Matta-Clark, les responsables de ces refabrications ont été attentifs à réactualiser son caractère performatif tel que l'avait conçu l'artiste. Ils pouvaient aussi se référer au film qui constitue un document pouvant servir de script de l'œuvre.

Ainsi, le New Museum (New York) a eu recours au savoir oral et pratique des collaborateurs de Matta-Clark pour réaliser la première refabrication d'Open House présentée, en mai 1981, dans l'exposition intitulée Alternatives in Retrospect, an Historical Overview 1969-1975. Richard Nonas avait été désigné par le Gordon Matta-Clark Trust comme représentant des ayants droit de l'artiste et responsable des refabrications de l'œuvre. En collaboration avec Jene Highstein et Ted Greenwald, il a refabriqué Open House en prenant pour modèle sa première version et en demeurant fidèle à son principe de fabrication résultant du recyclage des matériaux prélevés dans un chantier de démolition. De plus, par sa présentation hors les murs du musée, devant le 65 de la $5^{e}$ avenue, le New Museum a réactualisé ses conditions de mise en vue.

Lors de sa deuxième refabrication en 1985 par le Museum of Contemporary Art de Chicago qui présenta Cordon MattaClark: a Retrospective, cette institution muséale, dans le but de garantir l'authenticité de cette occurrence de l'œuvre, a introduit un nouvel outil de médiation par la production du premier script écrit de l'œuvre. Cette exposition étant itinérante et l'exemplaire de Chicago ne voyageant pas, ce document visait à transmettre aux musées hôtes de l'exposition des instructions très précises destinées à guider leur propre refabrication. Il est constitué d'un texte descriptif des composantes de l'œuvre, d'un diagramme foumissant de l'in- 
48

Archives Museum of Contemporary Art, Chicago (Copyright Material, Museum of ContemporaryArt of Chicago Not for publication). Installation Manual. formation sur les dimensions de l'espace intérieur et des photographies de l'exemplaire présenté à Chicago. On y lit qu'elle doit être à l'identique de cet exemplaire qui a pour modèle la première version d'Open House. Les règles à respecter concernent tant le processus de fabrication que les composantes matérielles de l'œuvre: elles prescrivent de louer une benne à ordure, non dotée d'un " toit " et ayant déjà été utilisée, d'utiliser des matériaux de recyclage, de diviser l'espace intérieur par la construction de cloisons et l'insertion de neuf portes. Toutefois, le nombre de pièces compartimentant l'intérieur de "l'habitation" peut être différent de l'exemplaire présenté à Chicago ou de la première version. Enfin, il est recommandé de présenter l'œuvre à l'extérieur du musée ${ }^{48}$. Le Museum of Contemporary Art de Chicago avait réactualisé son caractère urbain et contextuel en la présentant sur le trottoir, devant le musée, durant la journée du 9 mai.

Ce mode de mise en vue a été celui des itérations d'open House jusqu'à la fin des années 1980 . Elle a cependant été exposée dans l'espace intérieur de la ACE Gallery à Los Angeles, en 1989, dans le contexte de l'exposition Entering into the Sculpture. Peu de temps après, l'exemplaire du Musée d'art contemporain de Chicago a été mis sur le marché de l'art par les ayants droit de l'artiste. Il a été acquis par le collectionneur Daniel Varenne et mis en dépôt par ce dernier au Musée d'art moderne et contemporain de Genève, en 1994 .

L'œuvre était accompagnée du certificat d'authenticité rédigé par le Gordon Matta-Clark Trust. Ce document attribue à l'exemplaire acquis les valeurs d'unicité et d'authenticité traditionnellement légitimées par le système des beaux-arts. Il déclare qu'Open House se manifeste sous la forme d'une œuvre permanente et pérenne qu'il qualifie de final artwork et à laquelle il attribue une identité numérique. Sa valeur d'unicité est confirmée par cette déclaration qui dit que le Cordon Matta-Clark Trust ne reconnaît que cet exemplaire comme étant authentique et originellement créé par Matta-Clark, le classant ainsi dans la catégorie de la réplique dont la production a été déléguée aux 
ayants droit de l'artiste. ${ }^{49}$ Par ailleurs, il assure la pérennité des conditions originelles de mise en vue de l'œuvre en autorisant deux modalités d'immanence d'Open House : celle de l'œuvre dotée d'une matérialité pérenne et celle de l'exemplaire produit pour une exposition, dénommé Temporary Installation Work. Sa fabrication doit être autorisée par le propriétaire de l'exemplaire pérenne et le Gordon MattaClark Trust. Sa durée ne doit pas aller au-delà de trois mois. Et le certificat préserve l'unicité de l'exemplaire pérenne en précisant qu'il ne doit pas être présenté simultanément à cette exposition temporaire. Au cours de la décennie 1990, des exemplaires temporaires d'Open House ont été présentés dans des expositions tenues au Centre Julio Conzalez, Valence, du 3 décembre 1992 au 31 janvier 1993, et à PS1 à New York à l'été 1998. Ces refabrications, qui avaient obtenu l'accord de son collectionneur, Daniel Varenne, ont été supervisées par Richard Nonas.

Afin de garantir l'authenticité des occurrences de l'exemplaire temporaire, le Gordon Matta-Clark Trust a également produit un script de l'œuvre, intitulé «Instructions for re-building the Gordon Matta-Clark Dumpster in the original steel container", qui sert dorénavant de guide aux refabrications de l'œuvre. On peut le lire comme résultant d'une interprétation approfondie des intentions originelles de Matta-Clark. On y trouve des informations très précises sur les composantes matérielles de la première version d'Open House et sur son processus de fabrication. Le document recommande aussi de ne pas enlever les traces du travail de construction, telles les esquisses tracées sur les murs par les menuisiers. Il insiste sur le fait que l'espace intérieur doit avoir un caractère non fini, que les murs de placoplâtre ne doivent pas être peints, que les clous des pentures des portes peuvent être laissés apparents : "it should be rough and crude" 50 . Afin de rendre encore plus manifeste le caractère non-luxueux, participatif et ludique d'Open House, on souhaite que les visiteurs écrivent ou dessinent des graffitis sur ses parois ou encore on invite les ouvriers ayant collaboré à sa refabrication à y inscrire leur nom.
49

Archives, Musée d'art moderne et contemporain de Cenève. Le certificat d'authenticité d'Open House. produit par le The Gordon MattaClarkTrust, dont les membres sont Anne Alpert, Jane Crawford et Joseph Kosuth.

5o

Archives, Musée d'art moderne et contemporain de Genève. Instructions for re-building the Cordon Matta-Clark Dumpster in the original steel container. Produit parThe Cordon Matta-Clark Trust. 
51

Archives, Musée d'art moderne et contemporain de Genève. Instructions for re-building the Gordon Matta-Clark Dumpster in the original steel container. Certificat d'authenticité accompagnant Open House, produit parThe Cordon Matta-Clark Trust.
Ce protocole concerne également le dispositif d'installation de l'exemplaire temporaire d'Open House dans une salle d'exposition, le script ne restreignant pas sa monstration dans un espace extérieur. Dans un tel contexte, la principale règle à suivre est de faciliter l'accès du public à l'espace intérieur de l'œuvre. On souligne, avec insistance, qu'Open House doit être offerte à son usage. Mettant en valeur le caractère non pérenne de l'exemplaire temporaire, le document recommande de ne pas se préoccuper de sa possible détérioration par les interventions des visiteurs:

It is supposed to get more and more worn. It is also supposed to be fun. If eventually it gets too bad, it can easily be re-built [...] wherever it is, this sculpture will get more and more rough and broken-down as time gres by. That is the way it should be. When it no longer feels like a cordon place and seems more like a garbage-pile, destroy it, or re-build it, and feel good about the whole process ${ }^{5}$.

La présentation d'Open House à New York en 2007, dans l'espace de la galerie David Zwirner, qui représentait le Gordon Martta-Clark Fund, dans le cadre de l'exposition Cordon Matta-Clark et de Rirkrit Tiravanija, constitue une application de ce protocole. Son caractère performatif avait été actualisé en mettant à la disposition des visiteurs des cannettes de peinture afin qu'ils puissent tracer des graffitis. Cette actualisation se manifestait aussi par les énoncés tracés sur les parois de la benne à ordure, car ils implantaient Open House dans le contexte socioculturel contemporain.

En 2008, le Musée d'art moderne et contemporain de Genève a acquis, du collectionneur Daniel Varenne, l'exemplaire pérenne d'Open House accompagné de son certificat d'authenticité. Cette acquisition constitue donc une nouvelle étape de la carrière de l'œuvre qui a donné lieu à l'attribution de deux dates de production par le musée: 1972, date de sa production originelle et de sa mise en vue inaugurale, et 1985 , date de production de l'exemplaire pérenne résultant de son exposition au Musée d'art contemporain de Chicago ; c'est cet exemplaire qui a été acquis par le musée. L'attribution de ces deux dates indique bien que le Musée d'art moderne et contemporain de Genève considère Open House comme une cuvre ouverte dont la production 
peut s'étaler sur différents moments de sa vie publique. Lors de son entrée au musée, l'exemplaire de Chicago a été l'objet de modifications matérielles : les graffitis et les marques, tels les chiffres, tracés sur la benne à ordure, ont été effacés et ses portes ont été remplacées par d'autres qui ont été prélevées dans un chantier de démolition à proximité du musée et elles se ferment dorénavant mécaniquement ${ }^{52}$.

Depuis la mort de l'artiste, ses ayants droit, en collaboration avec des acteurs du monde de l'art, ont donc modifié l'identité spécifique et numérique originelle d'Open House, puisqu'elle se manifeste dorénavant selon deux modalités : celle de l'œuvre allographique, dont la durée de matérialisation est celle de son exposition, et celle de la réplique dotée d'une matérialité pérenne qui n'est pas celle de son état originel, mais qui renvoie au moment de sa production dans le cadre de la rétrospective de Matta-Clark au Musée d'art contemporain de Chicago. L'authenticité de ces deux types d'exemplaires, par ailleurs, est fondée sur l'application des principes originels de fabrication de la première version d'Open House qui ont été institutionnalisés par le document rédigé par le Gordon MattaClark Trust. C'est dorénavant le Musée d'art moderne et contemporain de Genève qui est responsable de la pérennité de ce caractère polymorphe de l'immanence d'open House, pour reprendre les termes de Genette, ou de ses deux modes de manifestation.

\section{La manipulation des conventions muséales}

Cette analyse a mis au jour des modes de manipulation des conventions du système traditionnel des beaux-arts visant la valorisation de la singularité et de l'unicité des œuvres acquises. Ainsi, en effectuant le passage de Moon Is the Oldest TV vers les arts reproductibles, Nam June Paik a produit une nouvelle œuvre dotée d'une identité spécifique et numérique qui la différentie de l'œuvre acquise par le MNAM, dont l'unicité et la singularité sont fondées sur son caractère performatif. Par ailleurs, notre étude a également démontré que l'ordre hiérarchique de la doxa muséale différentiant le premier exemplaire de la réplique et de la
52

Entretien de Sophie Costes, conservatrice, Musée d'art moderne et contemporain de Genève, avec Véronique Rodriguez, 18 juin 2009 
copie d'exposition est le plus souvent objet de négociations entre l'artiste, ou ses ayants droit, et l'institution muséale. La catégorie copie de prêt ou copie de voyage, qui conjugue des caractéristiques de la réplique et de la copie d'exposition, est souvent utilisée par les professionnels des musées pour classer le deuxième exemplaire d'une œuvre collectionnée. Son caractère pérenne la distingue de la copie d'exposition; et son appellation indique qu'elle est présentée hors les murs de l'institution collectionneuse et garantit ainsi sa fonction d'exposer seule l'œuvre originelle. Cette classification permet aussi de préserver la singularité et l'unicité de l'œuvre acquise, ou de sauvegarder sa référence à l'acte originel de sa création, et d'adapter les valeurs du système traditionnel des beaux-arts à une nouvelle situation mise en place par les œuvres contemporaines. Elle a été appliquée par le Musée des beaux-arts du Canada à la fixation du statut de la réplique de Untitled (1967-1968), produite par Robert Morris, ainsi que par le Musée d'art moderne (Centre Pompidou) à celui du deuxième exemplaire de Salle blanche de Marcel Broodthaers dont la production a été initiée par l'ayant droit de l'artiste. Dans le cas de ce dernier exemple, cette opération va cependant à l'encontre de l'ordre hiérarchique proposé au colloque The Replica and its Implication in Modern Sculpture tenu à la Tate Callery en 2007 qui classait l'exemplaire produit par les ayants droit dans la catégorie du document ou ne lui accordait qu'une fonction informative. Le MNAM a, au contraire, reconnu la délégation auctoriale de l'ayant droit de l'artiste. Nous avons également relevé ce phénomène dans le cas de la production de l'exemplaire pérenne d'Open House de Gordon Matta-Clark, dont les représentants ont fixé deux modes d'immanence de cette œuvre: celui de son régime allographique originel et celui de l'exemplaire pérenne produit sous leur supervision. Cette adaptation du musée à un double mode de manifestation des œuvres de sa collection est aussi représentée par la réexposition de Les Piques d'Annette Messager qui, durant le temps d'une exposition, a attribué la fonction d'élément dans un ensemble à deux exemplaires de cette série. Cette situation a aussi été observée dans le cas de Léviathan main toth d'Ernesto Neto, dont le protocole d'acquisition fixé par le Fonds national d'art contemporain et 
l'artiste lui alloue un double statut, car elle peut être exposée comme une œuvre autonome et comme une partie de Léviathan Thot. Ces cas illustrent l'instauration, le plus souvent par contrat, d'un partenariat professionnel liant l'institution collectionneuse à l'artiste ou à ses ayants droit. Ce modèle a été aussi mis en place par les œuvres allographiques dont l'énoncé verbal est porteur de l'identité spécifique et numérique de l'œuvre et qui met ainsi en cause l'idée d'exemplaire originel. Dans notre corpus, Toile à l'unité / Légende de Claude Rutault est une figure typique de ce phénomène.

Tous ces cas de figures analysés illustrent bien la mutation de la culture muséale qui participe ainsi à l'instauration d'un nouveau régime artistique dont les paramètres ne sont sans doute pas explicitement formulés, mais qui est déjà, en partie, établi. 
Summary

Translated by Sophia Arenzon

Variability, the Specific and numerical Identities of Contemporary Artworks
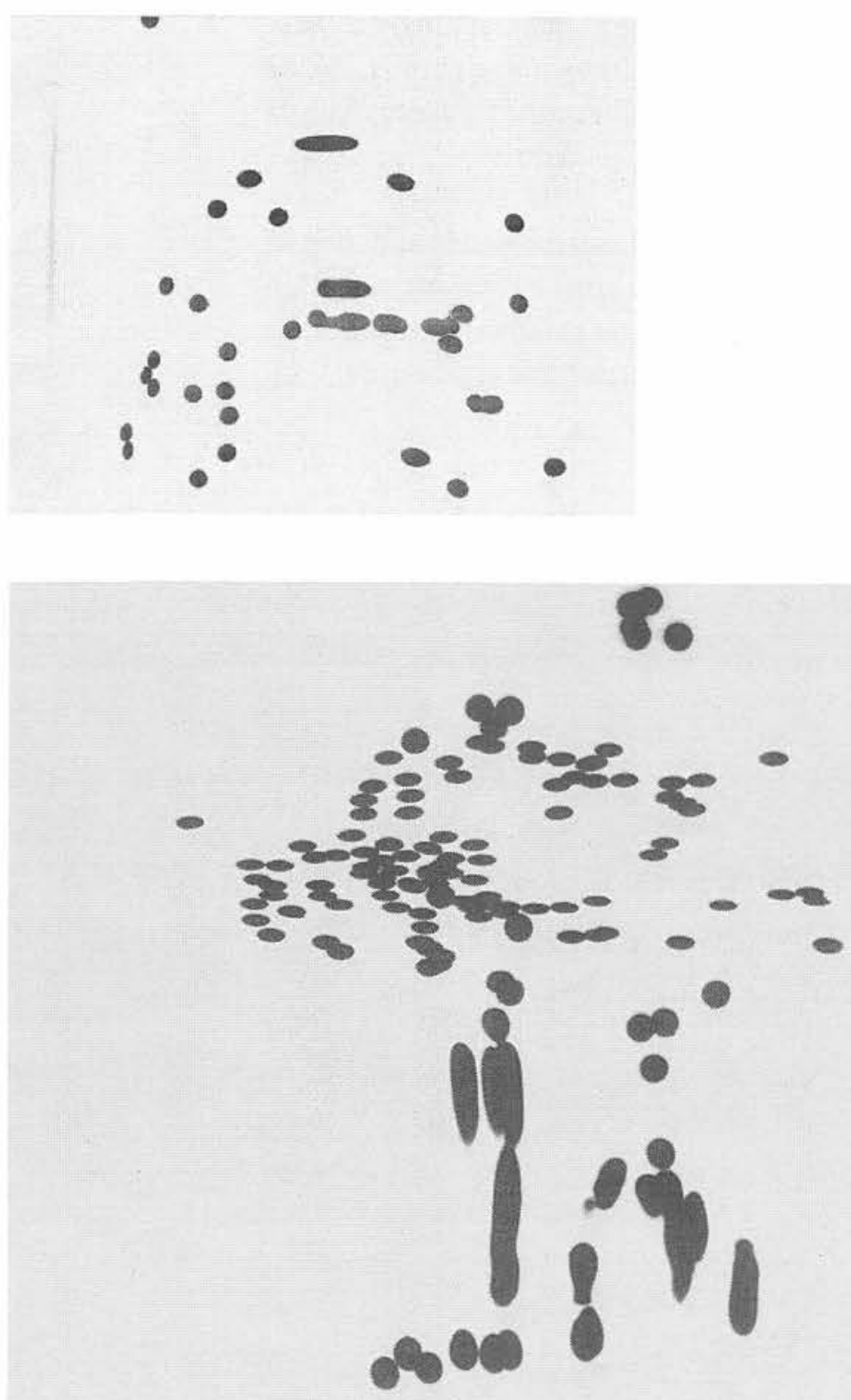
Re-exhibiting a contemporary work of art can produce a new copy of a work that was conceived as unique. The present article examines the ways a museum deals with this situation. which questions its conventions regarding the preservation of the work's specific identity as well as its numerical identity or uniqueness. Luis Prieto has found a strong link between these two characteristics of the work of art, noting that the artistic institution considers the original work to be numerically determined because it refers to the act of its creation, which gives it its specific identity. Moreover, Gérard Genette has emphasized that works of art can appear in a mode of plural immanence. For instance the artist can produce a replica that, artistically speaking, complements the original work; or an exhibition copy of the same work can be produced. By empirically analysing such cases we will see that their classification. in relation to the original work, most often results from negotiations between the museum and the artist or copyright holders. We will also analyse the case figure of the re-exhibition, which presents one or several elements of an already produced work as a work of its own. This case figure introduces the following question: how does the artist and the museum professionals recognize the material and conceptual integrity of the original work? Finally we will examine how the museal institution established a framework to manage the contemporary works acquired as a spoken word that bears the uniqueness and singularity of the work. Unlike the cases mentioned earlier, its various units lack a distinct specific or numerical identity because they result from the actualisation of this spoken word or of other documents that contain the elements constituting the ideal work of art. This category has helped moving the original materiality of the work towards its author by introducing the idea that the authenticity of its performance comes from the judgement of the artist through these documents. Possessing such an authority can also lead the artist or prompt his copyright holders to modify the specific identity of an allographic work of art by granting material permanence to one of its copies. We will also analyse a case figure for this transformation mode. This article will demonstrate that contemporary works of art manipulate museal conventions and help establishing a new artistic regime. 